\title{
Risk Assessment of Obstacle Collision for UAVs Under Off-nominal Conditions
}

\author{
Portia Banerjee ${ }^{1}$, George Gorospe ${ }^{2}$ \\ ${ }^{1,2}$ KBR Inc., NASA Ames Research Center, Moffett Field, 94035 CA, United States \\ portia.banerjee@nasa.gov \\ george.gorospe@nasa.gov
}

\begin{abstract}
Enabling operations of unmanned aerial vehicles (UAVs) in low-altitude airspace demands the need of robust risk monitoring framework for assessing the safety of airspace, groundstructures and people. As widespread applications emerge, the need of risk assessment becomes increasingly important for UAV flights beyond visual line-of-sight, especially subjected to off-nominal conditions introduced by component failures, degraded controllability or environmental disturbances such as wind gusts in an urban canyon. From a safety perspective, collision with obstacles can be detrimental not only to the vehicle and payload, but also to the structure and people on ground. Although it is safe to assume that approved UAVs would be equipped with collision avoidance systems, risk of collision which can be predicted for a flight trajectory even before the UAV encounters any obstacle is beneficial to resolve contingencies in its decision-making under off-nominal conditions. In this paper, a framework is presented for computing the risk of collision with obstacle based on a UAV's predicted trajectory, proximity to static and dynamic obstacles, sub-system state-of-health and external wind conditions. The conditional probability of trajectory deviation is generated using a Bayesian Belief Network (BBN) based on on-board sensor measurements. Further, a kinematic 3-DOF model is implemented to compute deviation in UAV's trajectory subjected to one case study of off-nominal condition i.e. wind gusts. Finally, the integrated risk factor is demonstrated on real data from experimental flights of an octocopter at NASA Langley Research Center, in presence of simulated obstacles and wind conditions. The proposed approach would enable risk-informed decision making process for timely mitigation of current and future unsafe events.
\end{abstract}

\footnotetext{
Portia Banerjee et al. This is an open-access article distributed under the terms of the Creative Commons Attribution 3.0 United States License, which permits unrestricted use, distribution, and reproduction in any medium, provided the original author and source are credited.
}

\section{INTRODUCTION}

Airspace is anticipated to undergo an enormous transformation in the future, with the incorporation of unmanned aerial vehicles (UAVs) (Kopardekar et al., 2016; FAA, 2018), both in commercial and military sectors. Owing to the substantial benefits of UAVs in multiple application areas such as infrastructure monitoring, delivery of goods, precision agriculture, public safety, search and rescue, disaster relief and weather monitoring, all sectors of industry, academia, and the government have been working towards a seamless integration of sUAS into the National Airspace System (NAS) (National Academies of Sciences, Medicine, et al., 2018). The final goal of the technology development is to enable UAV flights in low-altitude airspace (upto $500 \mathrm{ft}$ ) as well as beyond visual line of sight (BVLOS) operations over urban environment to extract full potential of such operations.

However, commercial implementation of these vehicles into the airspace has been relatively slow due to high risks associated with them. In order to enable wide-spread UAV operations in the airspace, it is imperative to demonstrate safety of such operations. Hazards need to be identified in-time and appropriate risk mitigation procedures need to be executed in the UAV integration. Currently, small UAV design does not require high reliability standards (King, Bertapelle, \& Moses, 2005; Freeman \& Balas, 2014), suggesting that failure rates of sub-systems and components during operations may be substantially higher than what has been seen in traditional manned aviation. Small UAVs have also intrinsic limitations due to their size and cost. They cannot carry heavy or expensive on-board sensors, computing systems or hardware for large-bandwidth communications with the ground station, thus limiting the ability to perform self-diagnosis and prognosis. For those same reasons, batteries installed in those small vehicles are relatively small, and the discharge rate during flight may be faster than expected due to environmental conditions like temperature or wind. Further, lightweight vehicles suffer external disturbances like wind gusts and turbulence more frequently than larger aircrafts. It is known that 
wind tunnel effects caused by buildings or other obstacles in urban environment may jeopardize vehicle stability, but they are extremely challenging to model and therefore may not be accurately predicted in-time.

Safety monitoring frameworks similar to the ones developed for manned aircraft (Roychoudhury et al., 2015, 2016; Spirkovska, Roychoudhury, Daigle, \& Goebel, 2017) may enhance unmanned traffic management with in-time monitoring and prediction tools for low-altitude operating vehicles. NASA's Aeronautics Research Mission Directorate (ARMD) strategic plan directs the development of advanced in-time safety assurance tools that can monitor, assess and mitigate risks for UAV operations (Aeronautics \& Administration, 2017). Under that initiative, several studies have been directed to prediction of future trajectory (Corbetta, Banerjee, Okolo, Gorospe, \& Luchinsky, 2019; Banerjee \& Corbetta, 2020), estimation of remaining battery life (Daigle \& Kulkarni, 2016), estimation of vibrational anomalies (Banerjee, Okolo, \& Moore, 2020) as well as assessment of risk to population on ground in the event of a crash (Ancel, Capristan, Foster, \& Condotta, 2019). Weibel \& Hansman (Weibel \& Hansman, 2004) and Clothier et al (Clothier, Walker, Fulton, \& Campbell, 2007) estimated expected rate of casualties per flight hour based on population density, area of exposure and failure rate. Kim (Kim, 2019) conducted a third-party risk assessment study for small UAVs in the event of a midair collision with another UAV. In all such studies, failures have been comprehended as an event leading to uncontrolled descent or collision that affect the population on ground directly. However, failure may not necessarily lead to a crash. Off-nominal events such as sudden wind gusts or degraded motor can cause vehicle instability forcing it to deviate from its planned path and lead to hitting obstacles. To the best of our knowledge, risk assessment to obstacle collision is relatively unexplored in the literature.

Many researchers have discussed frameworks for contingency plans when UAV encounters a dangerous situation during flight such as UAV lost communication link or fuel is low and cannot maintain the original planned path. In these, the operator has to provide fuel status, weather status or engine status to the decision making system which then creates casebased reasoning (Zhou \& Kwan, 2018; Pastor, Royo, Santamaria, Prats, \& Barrado, 2012). However, none of these studies computes in-time risk-aware contingencies that considers vehicle health prognostics in the loop. Although it is safe to assume that approved UAVs would be equipped with collision avoidance systems, risk of obstacle collision which can be predicted for a flight trajectory even before the UAV encounters any obstacle is beneficial to flight planning as well as resolving contingencies in its decision-making with a higher prediction horizon.

In this paper, a risk-assessment framework is presented par- ticularly to compute risk of collision of UAVs with static obstacles. The risk likelihood and severity is defined followed by a few case studies demonstrating the effect of multiple factors on the risk severity. The case studies are represented on a real UAV flying in a simulated environment. .

\section{Risk Assessment Framework of OBstacle COllision}

In previous studies, third-party risk on the ground population has been described as a function of probability of crash or un-powered descent of the UAV caused by off-nominal conditions. For example, Clothier et al. (Clothier et al., 2007) described estimated number of casualties per flight hour as Eq. (1). $p_{\text {failure }}$ is the probability of failure of the UAV, $p_{l o c}$ is the probability of impacting at a specific location on ground defined in terms of a binomial distribution, $A_{\text {exp }}$ is the area of exposure on the ground, $\rho_{p}$ is the population density and $p_{c a s}$ denotes the probability of casualty is rate of fatality of a person being exposed to ground impact by the UAV.

$$
E_{c}=p_{\text {failure }} p_{\text {loc }} A_{\text {exp }} \rho_{p} p_{\text {cas }}
$$

Similarly Ancel et al. defined non-Participant casualty risk in terms of expected casualties on ground, as denoted in Eq. (2) (Ancel, Capristan, Foster, \& Condotta, 2017).

$$
E_{c_{j}}=\sum_{i=1}^{k} P_{I_{k}} A_{c_{j}} \rho_{p o p_{j}}
$$

where, $P_{I_{k}}$ is the probability of the UAV impacting the $k^{t h}$ grid cell on ground, $\rho_{\text {pop }}$ is the population density in the $j^{t h}$ sheltering unit obtained from survey data and $A_{c_{j}}$ is the area of casualty. $A_{c_{j}}$ is further dependent on the vehicle size, offnominal trajectory and impact point estimation. Additionally, the authors described the URAF methodology which uses a modified version of the risk matrix developed for un-manned aircraft systems, as cited within FAA's Safety Management System Manual (Administration, 2017) to establish and visualize real-time UAS flight risks. The rows in the matrix, as denoted in Fig. 1, represent mishap likelihood and the columns provide severity categories where intersection of the two signifies the associated risk to ground casualty.

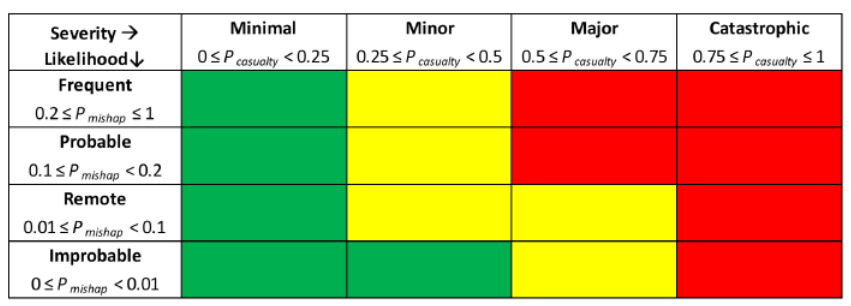

Figure 1. Notional risk matrix by FAA (Ancel et al., 2017). 


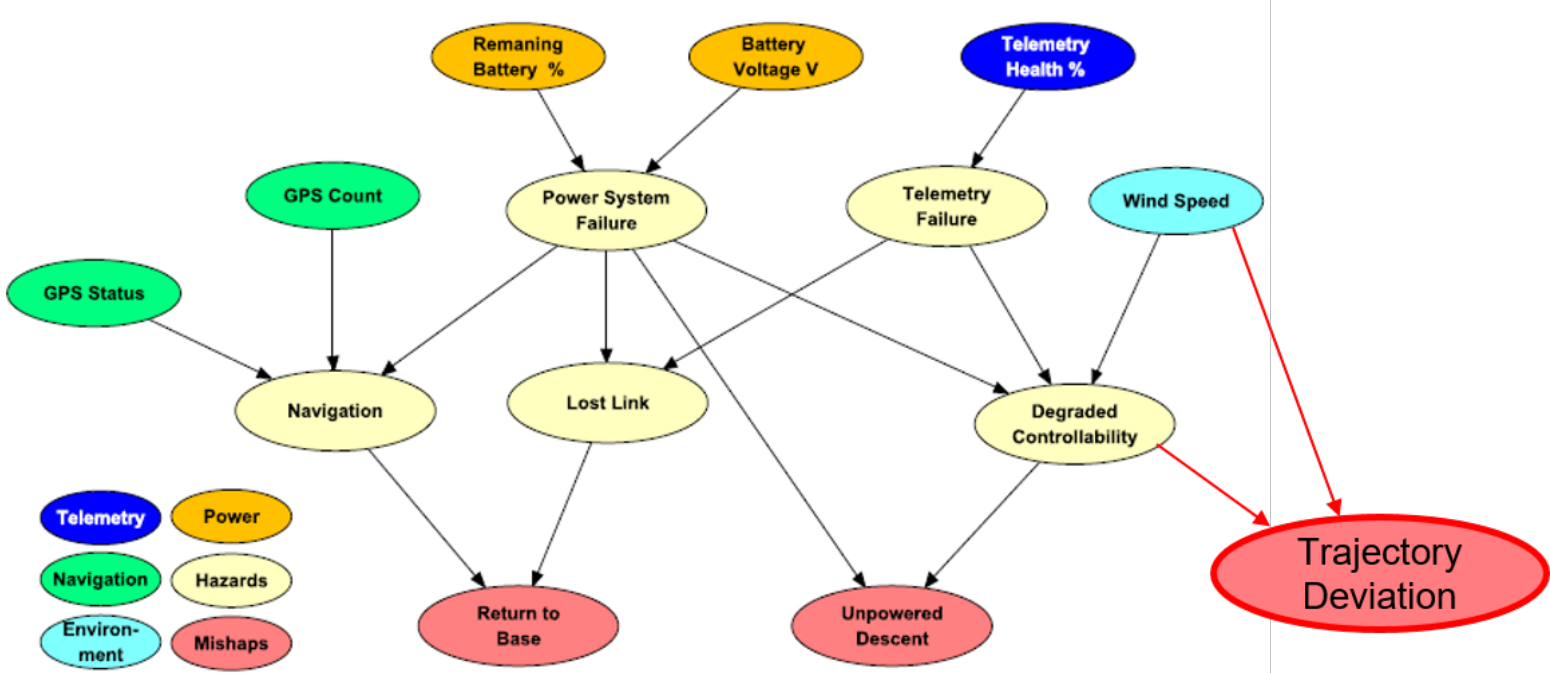

Figure 2. Bayesian Belief Network (Ancel et al., 2017) with additional node for trajectory deviation caused by wind or degraded controllability.

Based on the above formulations, likelihood of risk associated with obstacle collision for a UAV is defined as $p_{t r a j-d e v}$ or probability of trajectory deviation caused by any off-nominal event (wind gusts, degraded controllability etc.). This shall be computed using Bayesian Belief Network (BBN) that are most suited for extracting the causal effect between measurements and events and computing the conditional probabilities of events based on measured observations (Ancel et al., 2017). The BBN model continuously updates the input values for GPS Count, GPS Status, Remaining Battery, Battery Voltage, Telemetry Health, and Wind Speed dependent upon the aircraft altitude. These values are then used to calculate the probability of associated system failures, as depicted in figure 2 .

Further, severity of the risk $R I S K_{o b s}$ associated with obstacle collision is proposed in this paper as a function of probability of collision with obstacle $p_{c o l-o b s}$, area of the vehicle exposed to the obstacle when subjected to collision $A_{\exp }$ and probability that the location of collision represents an obstacle $p_{\text {obst }}$.

$$
R I S K_{o b s}=p_{\text {col-obs }} A_{\text {exp }} p_{\text {obst }}
$$

Intuitively, the area of exposure $A_{\text {exp }}$ is directly dependent on the size of the vehicle subjected to collision. On the other hand, $p_{\text {obst }}$ is computed based on the error associated with obstacle measurements. For a fixed obstacles such as trees and buildings, the obstacle locations and sizes are typically obtained from navigation sensors such as GNSS, IMU, LiDAR and cameras (Ilci \& Toth, 2020). If the error in the navigation measurements is assumed to adhere to a normal distribution

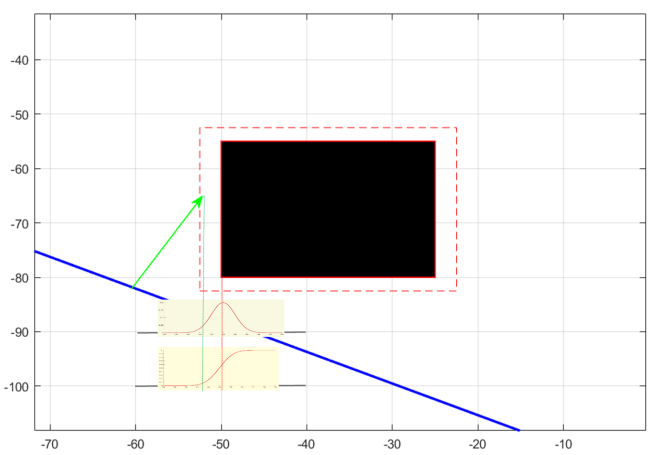

Figure 3. Schematic of $p_{\text {obst }}$ calculation.

with mean $\mu$ and variance of $\sigma^{2}$, the $p_{\text {obst }}$ can be defined as the cumulative distribution function, denoted in Eq. (4). Here $x$ is the position at which collision with the obstacle takes place, as shown in figure 3.

$$
p_{\text {obst }}=\frac{1}{2}\left[1+\operatorname{erf}\left(\frac{x-\mu}{\sigma_{o b s} \sqrt{2}}\right)\right]
$$

The other critical parameter in the risk severity formulation is the probability of collision $p_{c o l-o b s}$ which depends on a number of factors such as proximity of UAV from the obstacle, local wind field magnitude and direction as well as the state-ofhealth of the propeller unit. Both wind and degraded propeller capability of a UAV causes deviation of its trajectory from its desired flight path which may yield to collision with a surrounding obstacle. The formulation of collision probability, 
taking wind field parameters and state-of-health of vehicle, is described in the following section.

\section{Probability of obstacle collision}

In this study, the effect of wind on trajectory deviation is defined using simple kinematic equations on a vehicle point-mass model (Valeriy \& Ihor, 2018; D'Souza, Ishihara, Nikaido, \& Hasseeb, 2016). The vehicle state variables considered are the UAV airspeed $V$, heading $(\chi)$ determined by the directional path from one waypoint to another and flight path angle $(\gamma)$. The 3D coordinates of each waypoint is defined by its longitude $(\theta)$, latitude $(\phi)$, and altitude $(h)$. The

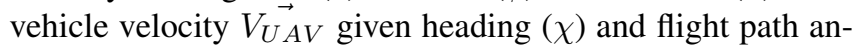
gle $(\gamma)$ is given by:

$$
\overrightarrow{V_{U A V}}=V \cos \gamma \cos \chi \hat{i}+V \cos \gamma \sin \chi \hat{j}+V \sin \gamma \hat{k}
$$

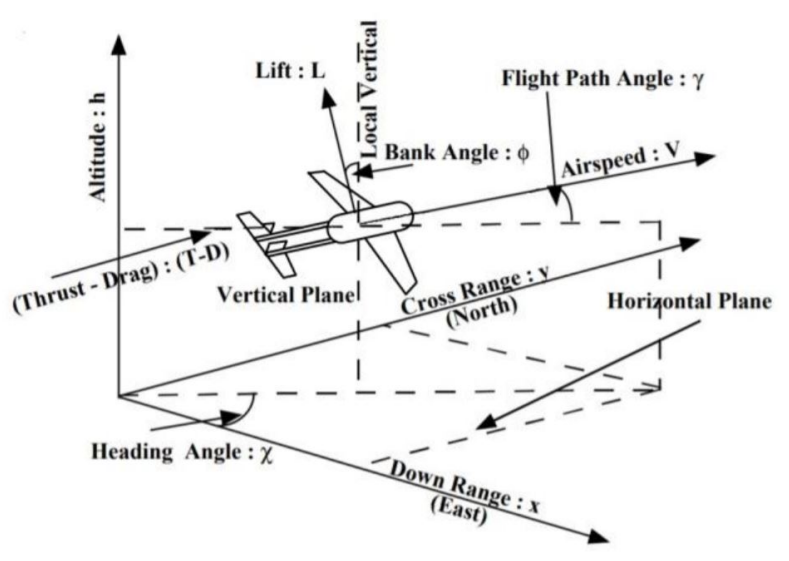

Figure 4. UAV coordinate system (Valeriy \& Ihor, 2018).

Considering wind velocity vector as $\vec{V}_{\text {wind }}$ and obtaining vehicle's cruise speed $\overrightarrow{V_{U A V}}$ from Eq. (5), the total vehicle velocity is given by $\vec{V}_{\text {total }}$.

$$
\begin{gathered}
\vec{V}_{w i n d}=U \hat{i}+V \hat{j}+W \hat{j} \\
\vec{V}_{\text {total }}=V_{U A V}+\vec{V}_{\text {wind }}
\end{gathered}
$$

The flight path angle is defined in the angle between the direction at which the vehicle's nose is pointing and the course over ground, as shown in Figure 4. Assuming the vehicle's flight path angle $(\gamma)$ is zero under no-wind condition, any non-zero value of $\gamma$ arises due to the vertical component of the wind speed. Hence the induced flight angle $\gamma_{\text {wind }}$ caused by wind is given by equation (8).

$$
\gamma_{w i n d}=\arctan \frac{W}{V \cos \gamma+U}
$$

Similarly, the horizontal deviation of the vehicle changes its heading $(\chi)$ and is caused by the horizontal components of the wind. Given the estimated time from detection of distur- bance on the vehicle to its recovery to original trajectory is denoted by $t_{\text {control }}$, the new position of the UAV deviated by wind $\left(\theta_{d e v}, \phi_{d e v}, h_{d e v}\right)$ at every position in the original reference trajectory $\left(\theta_{r e f}, \phi_{r e f}, h_{r e f}\right)$ is given by Eqs. (9)-(11). $t_{\text {control }}$ is dependent on UAV controller characteristics and state-of-health of its maneuver components.

$$
\begin{array}{r}
\theta_{\text {dev }}=t_{\text {control }} \frac{\left|\vec{V}_{\text {total }}\right| \sin \chi}{R \cos \phi_{\text {ref }}}+\theta_{\text {ref }} \\
\phi_{\text {dev }}=t_{\text {control }} \frac{\left|\vec{V}_{\text {total }}\right| \cos \chi}{R}+\phi_{\text {ref }} \\
h_{\text {dev }}=t_{\text {control }}\left|\vec{V}_{\text {total }}\right| \sin \gamma_{\text {wind }}+h_{\text {ref }}
\end{array}
$$

Collision with an obstacle occurs when the deviated position of the UAV $\left(\theta_{d e v}, \phi_{d e v}, h_{d e v}\right)$ hits or lies within the boundary of an obstacle. In practical applications, the wind velocity is measured or estimated at every point on the trajectory and be associated with stochasticity depending on the sensor characteristics and estimation process. Assuming Gaussian distribution of the wind field velocity is interpreted in terms of $n_{s}$ samples associated with probabilities, each deviated position in the trajectory is computed for $n_{s}$ samples, according to Eqs. (9)-(11). The samples which hit or lie within the boundary of any obstacle after $t_{\text {control }}$ seconds are identified as the collided samples $n_{o b s}$ and the probability of obstacle collision $p_{c o l-o b s}$ is defined in Eq. (3).

$$
p_{c o l-o b s}=\frac{n_{o b s}}{n_{s}}
$$

\section{RISK SEVERITY RESULTS: CASE STUDIES}

The risk of obstacle collision is computed for an experimental UAV flight conducted at the NASA Langley Research Center. Figure 5 (b) shows the flight plan executed with a DJI S1000 Octocopter in autonomous mode. The vehicle, as depicted in figure 5 (a), was equipped with Pixhawk autopilot hardware (http://pixhawk.org/) and commanded with Ardupilot software (http://ardupilot.org/) to fly through pre-defined 9 waypoints in 6 minutes.

Figure 5b shows the vehicle location "unrolled in time", calculated by the autopilot using GPS locations and its inertial measurement unit. The geospatial coordinates (latitude, longitude, and altitude) have been converted into a local, Earthfixed reference frame with origin at the take-off location, $x-$ axis pointing East, $y$-axis pointing North, and z-axis pointing upwards, forming a East-North-Up (ENU) reference frame. The z-axis represents altitude of the flight relative to its starting location which was from the top of a building. The UAV landed on the ground at certain times during the flight which are hence represented by the negative $\mathrm{z}$-values.

For demonstration of risk of obstacle collision, the above flight data is used in a playback mode with simulated obsta- 


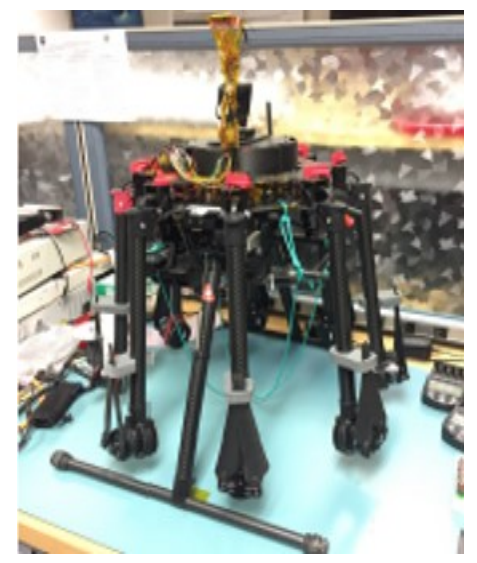

(a)
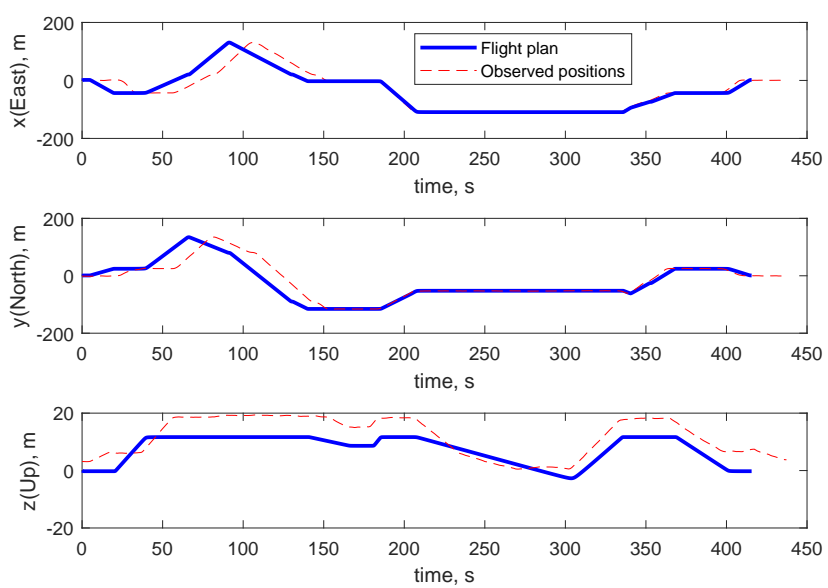

(b)

Figure 5. Example of DJIS1000 octocopter (5a) test flight in autonomous mode: the observed position is compared against the flight plan in a East-North-Up reference frame (5b).

cles, denoted by red squares and simulated wind field parameters. A 2D representation of the flight data is shown in fig 6 by considering the $\mathrm{x}$ and $\mathrm{y}$-axis data only. At first, based on the waypoint locations and commanded ETAs, the flight trajectory is generated using the B-spline algorithm (Corbetta et al., 2019). The blue vectors along the entire trajectory positions denote the vehicle airspeed under no-wind conditions.

\subsection{Case 1: Effect of wind field}

The magnitude and direction of the simulated wind-field are varied and the corresponding risk of obstacle collision is computed for the above UAV flight under different scenarios. At every position in the trajectory, the potential deviated positions are computed based on Eqs. (9)-(11) and the probability of collision with obstacles is generated by Eq. (12), depending on location of the obstacle with respect to the deviations. Figure 8 shows the 3 plots denoting risk of obstacle collision at different values of the wind magnitude $\left|V_{w}\right|$

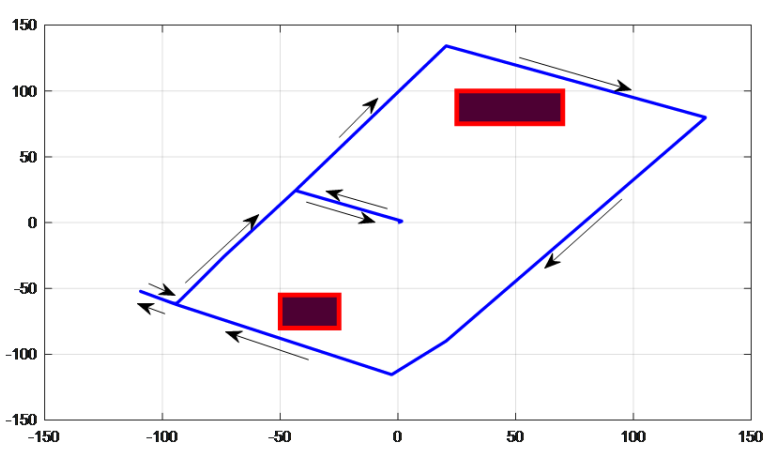

Figure 6. UAV flight trajectory in 2D representation simulated obstacles.

and direction $\theta_{w}$. In 2D representation, $\theta_{w}$ is defined as the angle between the resultant wind velocity vector and its $\mathrm{x}$ component. For better understanding, directions of the UAV and wind is shown in Figure 7.

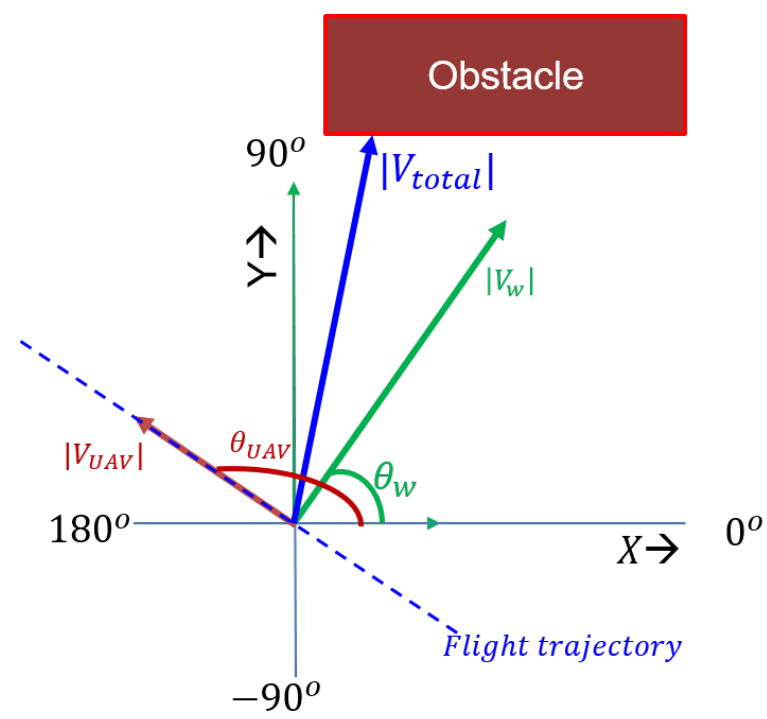

Figure 7. UAV flight trajectory in 2D representation simulated obstacles.

The normalized risk severity is plotted to compare the risks for 3 UAV flights of the same vehicle size, obstacle information and maneuverability conditions but subjected to different wind field. Depending on the intensity and direction of the wind acting at each point on the trajectory and the surrounding obstacle location, the deviated positions may or may not lead to a collision and hence the risk varies accordingly. For case 1, the magnitude of the simulated wind velocity is fixed at $\left|V_{w}\right|=\mathcal{N}(10,2) \mathrm{ms}^{-1}$ and direction $\theta_{w}=\mathcal{N}(40,5)^{\circ}$. As a result, the probability of collision with the obstacle 1 is higher resulting in higher risk severity (maximum value of 0.45 ) for the part of the trajectory close to it. When wind field direction is changed as in the 
$3^{\text {rd }}$ case where $\theta_{w}=\mathcal{N}(-90,5)^{\circ}$, the risk severity become high for the other part of the trajectory to the maximum of 0.8 . Magnitude of the wind velocity also affects the risk severity as can be observed from the risk plot in the $2^{\text {nd }}$ case with $\left|V_{w}\right|=\mathcal{N}(8,2) m s^{-1}$ and direction $\theta_{w}=\mathcal{N}(-120,5)^{\circ}$. The maximum value of the risk severity is less than 0.1 is this case.

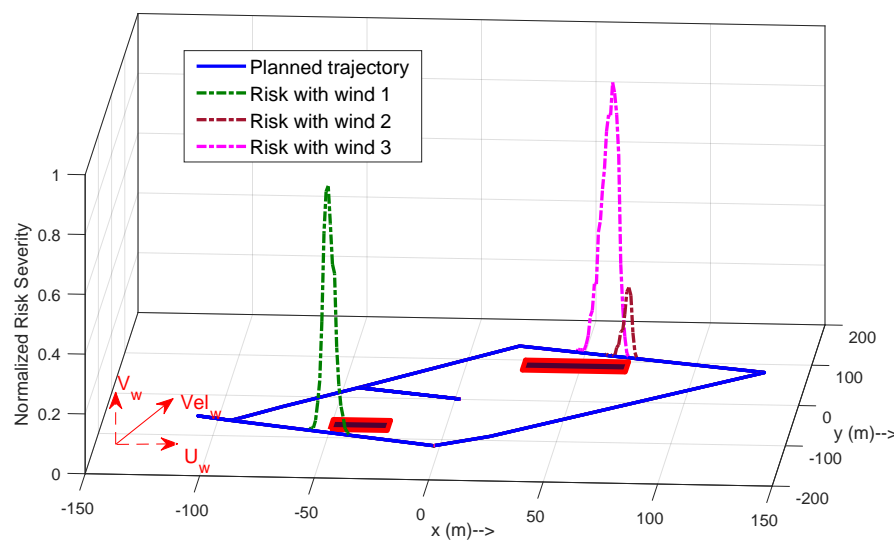

Figure 8. Effect of wind on Risk of obstacle collision. Wind 1: $\left|V_{w}\right|=\mathcal{N}(10,2) \mathrm{m} / \mathrm{s}, \theta_{w}=\mathcal{N}(40,5)^{\circ}$; Wind 2: $\left|V_{w}\right|=\mathcal{N}(8,2) \mathrm{m} / \mathrm{s}, \theta_{w}=\mathcal{N}(-120,5)^{\circ}$; Wind 3: $\left|V_{w}\right|=\mathcal{N}(14,2) m / s, \theta_{w}=\mathcal{N}(-90,5)^{\circ}$.

\subsection{Case 2: Effect of vehicle size}

Similar to wind direction, severity of risk to the obstacles is dependent on the size of the vehicle exposed to the obstacle. As denoted in Eq. (3), area of exposure during collision is higher for a larger vehicle and vice-versa, thereby associated with higher risk. UAVs are typically classified according to size that includes micro UAV, small UAV, medium UAV, and large UAV (Cano, Horton, Liljegren, \& Bulanon, 2017). The micro UAVs are extremely small in size and applies to sizes of about an insect to $30-50 \mathrm{~cm}$ long. The small UAV (sUAV) are UAVs with dimension greater than $50 \mathrm{~cm}$ and less than $2 \mathrm{~m}$. The medium UAVs have dimension ranging from $5 \mathrm{~m}$ to $10 \mathrm{~m}$ and can carry payloads of up to $200 \mathrm{~kg}$, while large UAVs include the ones mainly used for military operations or for future urban air mobility applications.

Figure 9 represents 2 plots denoting the risk severity for two of the most popular sUAVs in the market: 1) 3DR Iris and 2) DJI Phantom 2 flying over the same trajectory as defined in figure 6 (Cano et al., 2017). Both vehicles have been subjected to the same simulated wind field of magnitude $\left|V_{w}\right|=\mathcal{N}(14,2) \mathrm{m} / \mathrm{s}$ and direction $\theta_{w}=\mathcal{N}(-90,5)^{\circ}$. The size of the vehicle, defined in terms of motor-to-motor dimensions, is found to be $550 \mathrm{~mm}$ for the Iris and $350 \mathrm{~mm}$ for the Phantom UAV. As discussed, the normalized risk computed for the two cases based on equation (3) shows that higher risk is associated for the larger vehicle when exposed to the same wind in comparison to the smaller vehicle.

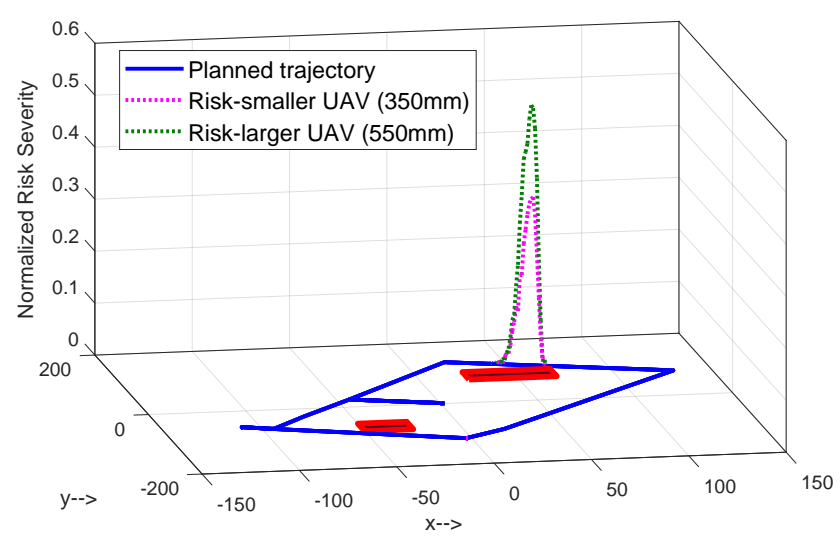

Figure 9. Effect of vehicle size on risk of obstacle collision (same wind conditions).

It should be noted that the current risk formulation lacks the penetration model which correlates the vehicle size and weight to the kinetic energy that will be transmitted to the buildings dependent on the material of the obstacle wall and roof properties. For example, a UAV hitting a glass building has higher associated risk to the building itself than the vehicle colliding with an asbestos roof. Such penetration models will be included in the future extensions of this study in order to refine the severity computation of the associated risk. Moreover, the risk comparison study assumes that the control time for both the vehicles is same, i.e., they take equal time to direct themselves back to the commanded trajectory after being deviated due to wind gusts. Such a generalization may not be valid for all categories of UAVs, since larger vehicles may be more capable of counteracting the wind effect and hence such inertia characteristics should be incorporated in the risk equation.

\subsection{Case 3: Effect of degraded maneuverability}

A faulty motor or failure in the propeller unit may yield to degraded capability in an UAV that may interfere with its maneuvering operations, therefore increasing the risk of collision both on ground as well as with obstacles. Diagnostics of a faulty condition during a UAV flight is beyond the scope of this study. However once a compromised component is identified, the risk of obstacle collision can be computed by increasing the nominal control time $t_{\text {control }}$. This assumption is based on the fact that a UAV with a faulty motor may generate reduced rotations-per-minute (RPM) that would result in reduced speed than a healthy vehicle. Under such condition, when the compromised vehicle is deviated due to an external disturbance such as wind, the controller would take higher time to recover and get back to the flight plan.

Figure 10 depicts two risk plots for a UAV under healthy and 


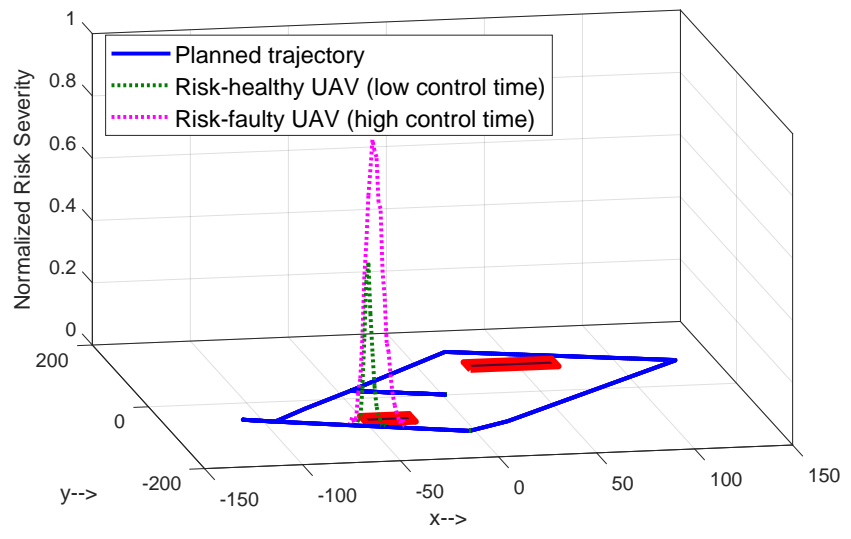

Figure 10. Effect of degraded maneuverability on risk of obstacle collision.

faulty condition with respective control time set at 1 and 1.5 seconds. As observed, the UAV with a faulty maneuverability condition is expected to be associated with higher risk of obstacle collision compared to its healthy counterpart. The wind field parameters are kept unchanged for both the cases with magnitude being $\left|V_{w}\right|=\mathcal{N}(8,2) \mathrm{m} / \mathrm{s}$ and direction being $\theta_{w}=\mathcal{N}(40,5)^{\circ}$.

Figure 11 demonstrates the benefit of risk assessment to select between two planned trajectories in the case of degraded capability. In Figure 11a, the two risk plots are associated with two trajectories that a healthy UAV can fly through following the same set of waypoints: (1) taking only right turns from start to end (clockwise direction) (2) taking only left turns from start to end (anticlockwise direction). When exposed to wind of magnitude $\left|V_{w}\right|=\mathcal{N}(8,2) \mathrm{m} / \mathrm{s}$ and direction $\theta_{w}=\mathcal{N}(90,5)^{\circ}$, the risk associated with the trajectory (1) was found to be higher than (2) in the case of the healthy $\mathrm{UAV}$, as observed in figure. Hence, the anticlockwise trajectory would be safer for the UAV under this event.

On the other hand, if one of the left motors is identified to be faulty while the UAV is in flight, the ability to complete left turns by the UAV will be compromised. Under this scenario if the UAV is subjected to the same wind field, the time of recovery $t_{\text {control }}$ will be higher than nominal operations which will eventually result in higher risk of obstacle collision. Figure $11 \mathrm{~b}$ denotes the risks associated with the same two trajectories for a UAV with degraded left maneuverability. In this case, the risk is higher for trajectory (2) and hence the clockwise trajectory would be safer for the UAV, unlike the previous case.

\subsection{Case 4: Effect of obstacle measurement noise}

Another important factor that may affect the risk severity is the noise associated with obstacle measurements. As mentioned in Section 2, the position boundaries of the obstacles

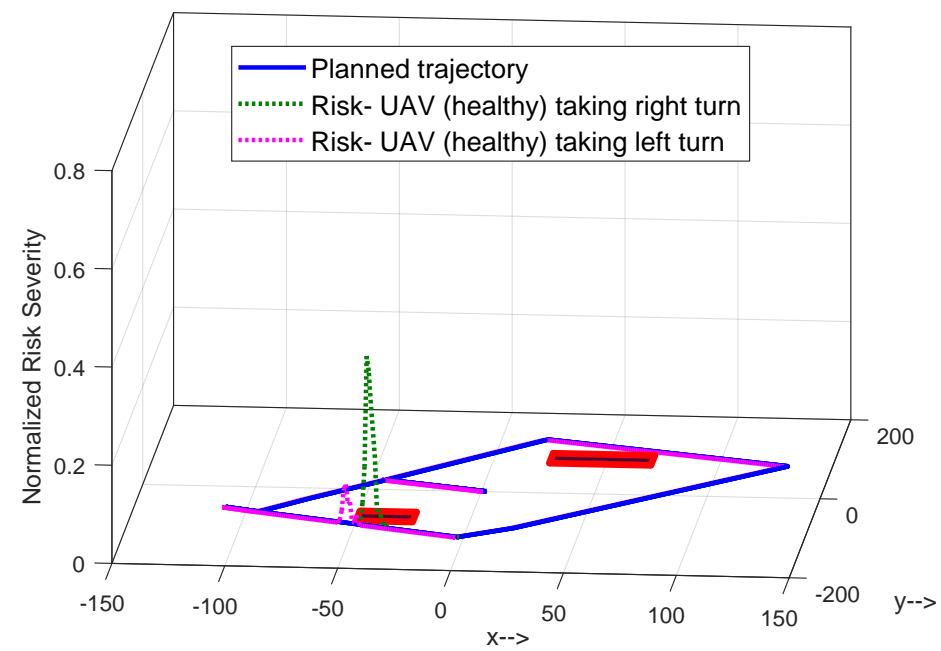

(a)

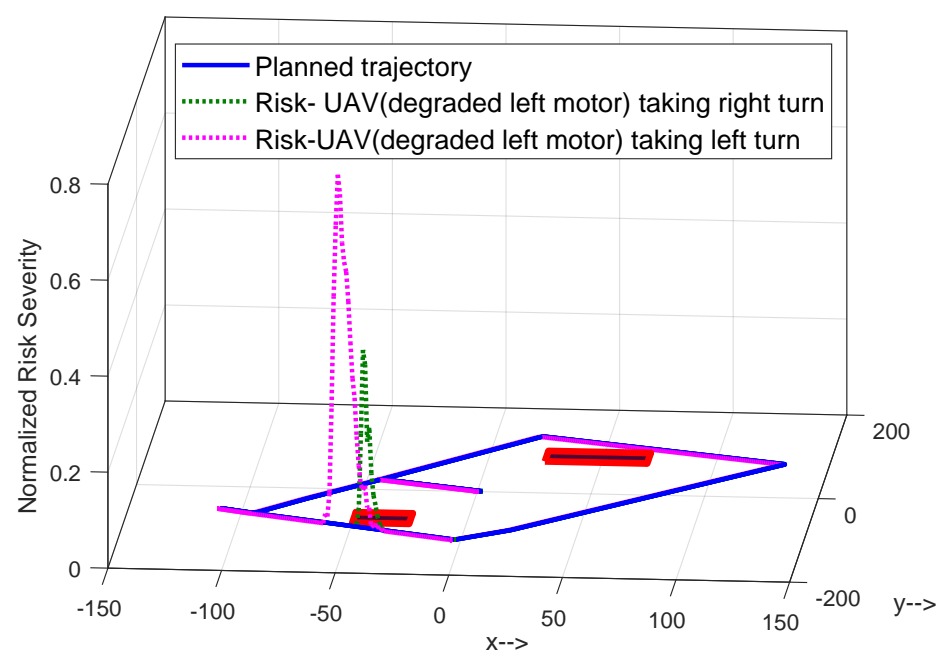

(b)

Figure 11. Risk of obstacle collision for clockwise and anticlockwise trajectories under same wind $\left|V_{w}\right|=\mathcal{N}(8,2) \mathrm{m} / \mathrm{s}$ and direction $\theta_{w}=\mathcal{N}(90,5)^{\circ}$ (a) Healthy UAV (b) UAV with degraded left maneuverability.

such as buildings and trees are obtained from navigation sensors including IMU, Lidar or cameras which are typically subjected to measurement noise. As a result, there is an inherent uncertainty associated with the point of collision representing a true obstacle. The risk of collision is therefore dependent on the measurement noise variance $\sigma_{o b s}$, as demonstrated in Figure 12. The two plots correspond to the risk severity for the same UAV flight exposed to the same wind conditions with magnitude $\left|V_{w}\right|=\mathcal{N}(6,2) \mathrm{m} / \mathrm{s}$ and direction $\theta_{w}=\mathcal{N}(90,5)^{\circ}$, but with different measurement noise variances of $\sigma_{o b s}=2$ and $\sigma_{o b s}=10$. For each case, the corresponding probability of the boundaries representing a true 
obstacle $p_{\text {obst }}$ is computed according to equation (4) followed bv the risk severitv obtained from (3).

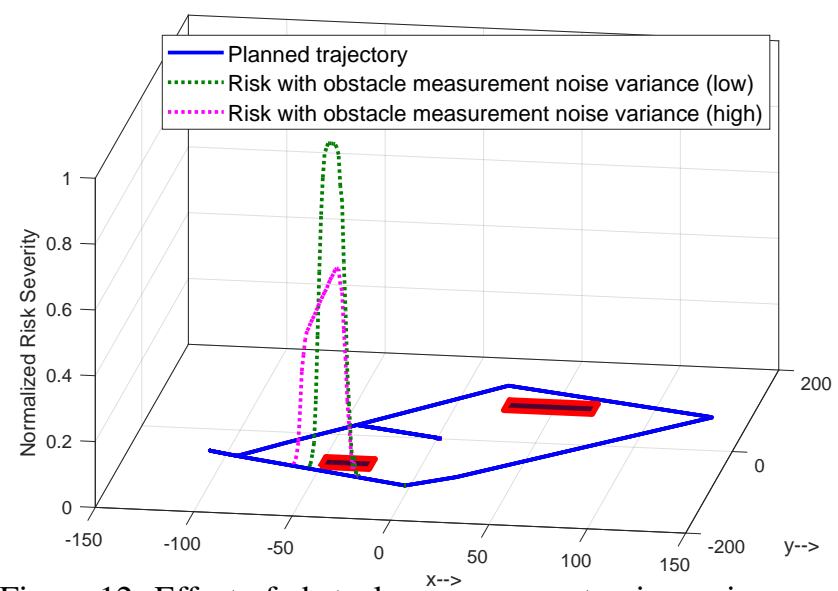

Figure 12. Effect of obstacle measurement noise variance on risk of obstacle collision (same wind conditions).

As denoted in Figure 12, it is observed that when the measurement noise is low, the probability of the obstacle boundary representing a true obstacle is high. Hence, the risk severity for a potential collision on hitting the obstacle is high. On the other hand, when $\sigma_{o b s}$ is high, the probability of the obstacle boundary representing a true obstacle is low. Hence the potential collision point caused by the deviated trajectory may not be a true obstacle which reduces the corresponding risk severity. However, since the measurement noise is high, a larger section of the trajectory has non-zero risk severity compared to the previous case of low $\sigma_{o b s}$.

\section{Conclusion}

In this paper, the risk of collision with an obstacle has been defined for an autonomous UAV. The risk likelihood is computed using bayesian network that generates the probability of trajectory deviation based on UAV health parameters such as GPS Count, GPS Status, Remaining Battery, Battery Voltage, Telemetry Health, and Wind Speed. Further, the risk severity is formulated as a function of multiple parameters including the probability of collision with obstacles, the area of exposure and obstacle measurement noise. The effect of individual parameters on the overall risk severity is demonstrated on a real UAV flight subjected to a simulated environment. The resultant risk plots integrates the effects of off-nominal conditions in a comprehensive fashion and hence enable decision making under such conditions. In the case studies reported in the paper, a stationary wind field has been assumed. However, the approach remains valid for local wind effects such as wind tunnel effects around obstacles in which case the wind velocity magnitude and direction will be different at different positions in the trajectory.

In this study, the probability of obstacle collision is dependent on the control time which is considered as a health indica- tor. In order to have low computation time, we have assumed empirical values of this parameter so far. Next, we need to investigate the change in vehicle dynamics for degraded controllability (e.g.: faulty motor). Besides, a point-mass model is currently assumed for the UAV which describes the kinematics of the vehicle. However, in order to generate more precise values of trajectory deviation caused by wind, wind effect needs to be incorporated into the vehicle dynamic model as well. Effect of uncertainty in vehicle navigation measurements will be incorporated further in order to compute the risk of obstacle collision in real time. Finally, the risk formulation will be used to examine safe distance requirements for a UAV flight flying through urban canyons and variable wind conditions.

\section{ACKNOWLEDGMent}

This work was supported by the System-Wide Safety (SWS) project under the Airspace Operations and Safety Program within the NASA Aeronautics Research Mission Directorate (ARMD).

\section{REFERENCES}

Administration, F. A. (2017). Safety management risk policy (faa order 8040.4b).

Aeronautics, N., \& Administration, S. (2017). Nasa aeronautics strategic implementation plan: 2017 update.

Ancel, E., Capristan, F. M., Foster, J. V., \& Condotta, R. C. (2017). Real-time risk assessment framework for unmanned aircraft system (uas) traffic management (utm). In 17th aiaa aviation technology, integration, and operations conference (p. 3273).

Ancel, E., Capristan, F. M., Foster, J. V., \& Condotta, R. C. (2019). In-time non-participant casualty risk assessment to support onboard decision making for autonomous unmanned aircraft. In Aiaa aviation 2019 forum (p. 3053).

Banerjee, P., \& Corbetta, M. (2020). In-time uav flighttrajectory estimation and tracking using bayesian filters.

Banerjee, P., Okolo, W. A., \& Moore, A. J. (2020). In-flight detection of vibration anomalies in unmanned aerial vehicles. Journal of Nondestructive Evaluation, Diagnostics and Prognostics of Engineering Systems, 1-8.

Cano, E., Horton, R., Liljegren, C., \& Bulanon, D. M. (2017). Comparison of small unmanned aerial vehicles performance using image processing. Journal of Imaging, 3(1), 4.

Clothier, R. A., Walker, R. A., Fulton, N., \& Campbell, D. A. (2007). A casualty risk analysis for unmanned aerial system (uas) operations over inhabited areas.

Corbetta, M., Banerjee, P., Okolo, W., Gorospe, G., \& Luchinsky, D. G. (2019). Real-time uav trajectory pre- 
diction for safety monitoring in low-altitude airspace. In Aiaa aviation 2019 forum (p. 3514).

Daigle, M., \& Kulkarni, C. S. (2016). End-of-discharge and end-of-life prediction in lithium-ion batteries with electrochemistry-based aging models. In Aiaa infotech@aerospace (p. 2132).

D'Souza, S., Ishihara, A., Nikaido, B., \& Hasseeb, H. (2016). Feasibility of varying geo-fence around an unmanned aircraft operation based on vehicle performance and wind. In 2016 ieeelaiaa 35th digital avionics systems conference (dasc) (pp. 1-10).

FAA. (2018). Unmanned aerial system (uas) traffic management (utm), concept of operations (Tech. Rep.). Federal Aviation Administration.

Freeman, P., \& Balas, G. J. (2014). Actuation failure modes and effects analysis for a small uav. In American control conference (acc), 2014 (pp. 1292-1297).

Ilci, V., \& Toth, C. (2020). High definition 3d map creation using gnss/imu/lidar sensor integration to support autonomous vehicle navigation. Sensors, 20(3), 899.

Kim, S. H. (2019). Third-party risk analysis of small unmanned aircraft systems operations. Journal of Aerospace Information Systems, 1-15.

King, D. W., Bertapelle, A., \& Moses, C. (2005). Uav failure rate criteria for equivalent level of safety. In International helicopter safety symposium, montreal (Vol. 9).

Kopardekar, P., Rios, J., Prevot, T., Johnson, M., Jung, J., \& Robinson, J. E. (2016). Unmanned aircraft system traffic management (utm) concept of operations. In Aiaa aviation forum.

National Academies of Sciences, E., Medicine, et al. (2018). Assessing the risks of integrating unmanned aircraft systems (uas) into the national airspace system. National Academies Press.

Pastor, E., Royo, P., Santamaria, E., Prats, X., \& Barrado, C. (2012). In-flight contingency management for unmanned aerial vehicles. Journal of Aerospace Computing, Information, and Communication, 9(4), 144-160.

Roychoudhury, I., Spirkovska, L., Daigle, M., Balaban, E., Sankararaman, S., Kulkarni, C., ... Goebel, K. (2015). Real-time monitoring and prediction of airspace safety (Tech. Rep.). National Aeronautics and Space Administration.

Roychoudhury, I., Spirkovska, L., Daigle, M., Balaban, E., Sankararaman, S., Kulkarni, C. S., ... Goebel, K. (2016). Predicting real-time safety of the national airspace system. In Aiaa infotech@aerospace (p. 2131).

Spirkovska, L., Roychoudhury, I., Daigle, M., \& Goebel, K. (2017). Real time safety monitoring: Concept for supporting safe flight operations. In 17th aiaa aviation technology, integration, and operations conference (p. 4494).

Valeriy, C., \& Ihor, S. (2018). Method of the multi-uav formation flight control. Archived Volume, 167.

Weibel, R., \& Hansman, R. J. (2004). Safety considerations for operation of different classes of uavs in the nas. In Aiaa 4th aviation technology, integration and operations (atio) forum (p. 6244).

Zhou, J., \& Kwan, C. (2018). A high performance contingency planning system for uavs with lost communication. In 2018 ieee international conference on prognostics and health management (icphm) (pp. 1-8). 